\title{
Mechanisms Linking the Gut-Muscle Axis With Muscle Protein Metabolism and Anabolic Resistance: Implications for Older Adults at Risk of Sarcopenia
}

\author{
Konstantinos Prokopidis ${ }^{*}$, Edward Chambers ${ }^{2}$, Mary Ni Lochlainn ${ }^{3}$ and \\ Oliver C. Witard ${ }^{4}$
}

' Department of Musculoskeletal Biology, Institute of Life Course and Medical Sciences, University of Liverpool, Liverpool, United Kingdom, ${ }^{2}$ Department of Metabolism, Digestion and Reproduction, Faculty of Medicine, Imperial College, London, United Kingdom, ${ }^{3}$ Department of Twin Research and Genetic Epidemiology, King's College London, London, United Kingdom, ${ }^{4}$ Faculty of Life Sciences and Medicine, Centre for Human and Applied Physiological Sciences, King's College London, London, United Kingdom

OPEN ACCESS

Edited by: Llion Arwyn Roberts, Griffith University, Australia

Reviewed by:

Andrea Ticinesi, University of Parma, Italy

Sergio Perez-Burillo, University of Granada, Spain

*Correspondence: Konstantinos Prokopidis k.prokopidis@liverpool.ac.uk

Specialty section: This article was submitted to Integrative Physiology, a section of the journal

Frontiers in Physiology

Received: 06 September 2021 Accepted: 07 October 2021 Published: 26 October 2021

Citation:

Prokopidis K, Chambers E, Ni Lochlainn M and Witard OC (2021) Mechanisms Linking the Gut-Muscle Axis With Muscle Protein Metabolism and Anabolic Resistance: Implications for Older Adults at Risk of Sarcopenia.

Front. Physiol. 12:770455.

doi: 10.3389/fphys.2021.770455
Aging is associated with a decline in skeletal muscle mass and function-termed sarcopenia-as mediated, in part, by muscle anabolic resistance. This metabolic phenomenon describes the impaired response of muscle protein synthesis (MPS) to the provision of dietary amino acids and practice of resistance-based exercise. Recent observations highlight the gut-muscle axis as a physiological target for combatting anabolic resistance and reducing risk of sarcopenia. Experimental studies, primarily conducted in animal models of aging, suggest a mechanistic link between the gut microbiota and muscle atrophy, mediated via the modulation of systemic amino acid availability and low-grade inflammation that are both physiological factors known to underpin anabolic resistance. Moreover, in vivo and in vitro studies demonstrate the action of specific gut bacteria (Lactobacillus and Bifidobacterium) to increase systemic amino acid availability and elicit an anti-inflammatory response in the intestinal lumen. Prospective lifestyle approaches that target the gut-muscle axis have recently been examined in the context of mitigating sarcopenia risk. These approaches include increasing dietary fiber intake that promotes the growth and development of gut bacteria, thus enhancing the production of short-chain fatty acids (SCFA) (acetate, propionate, and butyrate). Prebiotic/probiotic/symbiotic supplementation also generates SCFA and may mitigate low-grade inflammation in older adults via modulation of the gut microbiota. Preliminary evidence also highlights the role of exercise in increasing the production of SCFA. Accordingly, lifestyle approaches that combine diets rich in fiber and probiotic supplementation with exercise training may serve to produce SCFA and increase microbial diversity, and thus may target the gut-muscle axis in mitigating anabolic resistance in older adults. Future mechanistic studies are warranted to establish the direct physiological action of distinct gut microbiota phenotypes on amino acid utilization and the postprandial stimulation of muscle protein synthesis in older adults.

Keywords: anabolic resistance, sarcopenia, gut microbiota, dietary fiber, skeletal muscle, exercise 


\section{INTRODUCTION}

Sarcopenia is described as the age-related decline in skeletal muscle mass and function (Rosenberg, 1997) that was recently recognized as an independent geriatric condition (Cao and Morley, 2016) and is reported to affect $8-13 \%$ of older adults (Shafiee et al., 2017). Although aging is associated with a progressive decline in muscle mass and strength, an accelerated deterioration of muscle functional capacity has been observed in individuals with sarcopenia (Cruz-Jentoft et al., 2019). The clinical implications of sarcopenia include-but are not limited to-an increased incidence of falls and fractures, frailty, loss of mobility and independence, and premature mortality among older adults (Cruz-Jentoft et al., 2019). Hence, understanding the interplay between physiological mechanisms that underpin sarcopenia is fundamental to developing targeted and effective lifestyle approaches to reduce sarcopenia risk in our aging population.

Multiple physiological factors are proposed to underpin sarcopenia. These factors include-but are not limited to-agerelated changes in hormonal milieu (Sakuma and Yamaguchi, 2012), and gut physiology (Azzolino et al., 2019), a chronic state of low-grade inflammation (Beyer et al., 2012), insulin resistance (Cleasby et al., 2016), DNA damage, elevated oxidative stress, mitochondrial dysfunction (Jackson and McArdle, 2016), and suppressed satellite cell activity (Snijders et al., 2015), as reviewed previously (von Haehling et al., 2012). Ultimately, muscle atrophy is underpinned by a state of negative muscle protein balance whereby rates of muscle protein breakdown (MPB) exceed rates of muscle protein synthesis (MPS) over a given period of time. Of these two metabolic processes, there is general consensus that a diminished capacity for older adults to stimulate MPS, as opposed to an acceleration of MPB, mediates muscle atrophy with aging, at least in healthy individuals (Tipton et al., 2018). In this regard, whereas comparative studies of young and older adults have reported no clear differences in basal postabsorptive rates of MPS, an impaired response of MPS to ingestion of meallike quantities of protein (Guillet et al., 2004; Cuthbertson et al., 2005; Moore et al., 2015) and/or other anabolic stimuli such as resistance exercise (Kumar et al., 2009; Durham et al., 2010) have been consistently reported with advanced age. This metabolic phenomenon has been coined muscle anabolic resistance (AR) and is proposed to contribute to the progressive decline in skeletal muscle mass associated with aging.

The physiological mechanisms that mediate AR are multifactorial but, to this end, are not fully understood (Burd et al., 2012). Fundamentally, the diminished capacity for older adults to stimulate MPS is underpinned by a reduced systemic (Bohé et al., 2003) and/or intracellular availability of amino acids (Kimball and Jefferson, 2002). Physiological processes that contribute to this age-related decline in amino acid availability include an increased splanchnic retention of amino acids leading to reduced peripheral amino acid availability (Boirie et al., 1997), a reduction in amino acid transport to muscle tissue (Biolo et al., 1995), and an impairment in microvascular perfusion (capillary recruitment and dilation) (Phillips et al., 2015). Recent evidence also indicates an important role for the human gut microbiota environment in regulating the utilization of amino acids (Yatsunenko et al., 2012; Ticinesi et al., 2019). In this regard, gut microbiota dysbiosis (Mahnic et al., 2020) is a physiological phenomenon that describes an altered gut microbiota composition (Biagi et al., 2010; Odamaki et al., 2016) and diversity (Nagpal et al., 2018; Coman and Cristian, 2020), and is proposed as another mediator of age-related AR. Further evidence also exists that an altered gut microbiota may directly increase risk of sarcopenia through specific bacterial depletion and fecal transplantation (Liu et al., 2021). Hence, the aims of this opinion narrative review are two-fold. First, to offer hypothesis-driven insights into possible pathophysiological mechanisms linking gut microbiota dysbiosis with impaired skeletal muscle metabolism in older adults. Second, and based on limited existing evidence, to propose a series of potential, non-pharmacological, strategies targeted at combatting AR via modulation of the gut microbiota. Interventional approaches addressed in this narrative review are by no means exhaustive and are focused on dietary fiber consumption, probiotic and prebiotic supplementation, and resistance exercise training.

\section{GUT MICROBIOTA IN AGING}

The structure and diversity of the human gut microbiome plays a key regulatory role in physiological, metabolic, and immune function, and thus impacts human health and disease risk (Guinane and Cotter, 2013). Specifically, the gut microbiome contains millions of diverse microorganisms, termed gut microbiota, that modulate various metabolic pathways, including inflammatory gene expression, innate immune effector cells (i.e., monocytes, macrophages), glucose tolerance, and the release of gut hormones (Martin et al., 2019; Zheng et al., 2020). The multiple microbial phyla of the gut microbiome include Proteobacteria, Fusobacteria, Actinobacteria, Verrucomicrobia, Firmicutes (Clostridium, Enterococcus, Ruminococcus, Lactobacillus), and Bacteroidetes (Prevotella, Bacteroides), and account for the majority of the microbiota species present in the gut (Wang et al., 2020), although several bacterial species may be found in other organs including muscle, brain, liver, heart, and adipose tissue (Lluch et al., 2015).

The composition of gut microbiota is modulated by several factors including genetics, diet and physical activity levels (Milani et al., 2016; Ticinesi et al., 2017). Aging also is strongly associated with a decline in gut microbiome diversity species in the duodenum, jejunum, ileum, and colon (Sovran et al., 2019; Badal et al., 2020). Taxonomic differences during aging have been observed, namely that older adults are accompanied by higher levels of Bacteroides, Eubacterium, and Clostridiaceae, and decreased Bifidobacterium compared to young adults (Yatsunenko et al., 2012; Odamaki et al., 2016; Stewart et al., 2018). During the aging process, epithelial cell tight junctions are weakened (Lustgarten, 2016), decreasing the expression of intestinal epithelial tight junctions proteins (Tran and Greenwood-Van Meerveld, 2013). This disrupted intestinal barrier is linked to reduced intestinal motility and increased 
permeability, that are associated with higher levels of low-grade inflammation and immunosenescence that accompany various age-associated conditions (Belkaid and Hand, 2014; Bosco and Noti, 2021). Therefore, the various taxonomic changes that occur over time via altered microbial function and composition may affect immune and metabolic health with advancing age (Ling et al., 2020).

\section{THE GUT-MUSCLE AXIS IN SARCOPENIA}

Multiple lines of evidence from rodent studies suggest that the gut microbiota may be linked with sarcopenia. First, the microbiota of older mice was shown to exhibit an abundance of the Rikenellaceae family that is associated with an increased frailty index in a dose-dependent manner (Langille et al., 2014). Second, a higher Sutterella to Barneseilla ratio has been reported in older sarcopenic vs. healthy adult rats, corresponding with an altered inflammatory and immune profile and decline in triceps and gastrocnemius size (Siddharth et al., 2017). Third, germfree mice that lack the gut microbiota of pathogen-free mice exhibit a greater decline in skeletal muscle mass, quality and neuromuscular function compared to pathogen-free mice (Lahiri et al., 2019), despite having similar body weight (Hsu et al., 2015). Finally, antibiotic-treated mice were accompanied by increased muscle atrophy (Manickam et al., 2018; Nay et al., 2019; Okamoto et al., 2019), that was associated with microbial dysbiosis and inhibition of ileal fibroblast growth factor 15 (FGF15), whereas muscle atrophy was reversed following FGF19 treatment (Qiu et al., 2021). Hence, some mechanistic evidence exists in animal models that the gut microbiome may play a key role in physical performance, given that germ-free and antibiotic-treated mice express lower competence during muscle loading (Lahiri et al., 2019) and swimming time to exhaustion (Hsu et al., 2015; Huang et al., 2019), compared to pathogen-free mice.

The hypothesis that the gut microbiota may be linked with sarcopenia has also been examined in humans. Using $16 \mathrm{~s}$ RNA sequencing, a higher abundance of Lactobacillus and a reduction of Fusicantenibacter, Eubacterium, Lachnospira, Lachnoclostridium, and Roseburia genera was reported in sarcopenic patients compared with healthy controls (Kang et al., 2021). In addition, cross-sectional studies have revealed a higher ratio of Firmicutes/Bacteoidetes and lower overall microbial richness in older adult patient groups compared with healthy young adult controls (Mariat et al., 2009; Larsen et al., 2010; Claesson et al., 2012; Le Chatelier et al., 2013). Accordingly, a higher abundance of several bacteria, including Eggerthella, Bacteroides/Prevotella, Lactobacillus/Enterococcus, and a lower abundance of Enterobacteriaceae, Methanobrevibacter, and Akkermansia, have been observed in frail patient groups (Van Tongeren et al., 2005; Ponziani et al., 2021). Moreover, in sarcopenic and physically frail populations, an increased abundance of Oscillospira and Ruminococcus, and a decrease of Barnesiellacaea and Christensenellaceae taxa also have been reported (Picca et al., 2020). Similarly, sarcopenic patients displayed a significant reduction in Faecalibacterium prausnitzii,
Roseburia inulinivorans, and Alistipes shahii species that are all competent bacteria with prominent metabolic capacity in producing SCFA (Ticinesi et al., 2020). The 16S rRNA sequencing of human fecal samples from (pre)sarcopenic individuals showed a decline in Lachnospira, Fusicantenibacter, Roseburia, Eubacterium, and Lachnostrodium genera, and an increased LPS biosynthesis compared to healthy individuals (Kang et al., 2021). Taken together, these data indicate a link between the gut microbiota and muscle atrophy, and thus supports a gut-muscle axis hypothesis to explain, in part, skeletal muscle dysfunction during aging.

\section{GUT MICROBIOTA AND ANABOLIC RESISTANCE}

The gut microbiota also is proposed to play a causal role in AR (Frampton et al., 2020), as mediated by multiple inter-related physiological mechanisms (Figure 1). The causal mechanisms that underpin AR span several levels of physiology, including the gut, vascular system, and skeletal muscle (Burd et al., 2013). Altered gut microbiota composition during aging may be involved in oxidative stress, inflammation, and insulin resistance (Grosicki et al., 2018). An aging gut microbiota may increase intestinal permeability and LPS leakage from the intestinal lumen and cell membranes of gram-negative bacteria into the circulation (Maes et al., 2012; Alhmoud et al., 2019). Such modifications are associated with insulin resistance and increased inflammation (Liang et al., 2013; Choi et al., 2015; Moszak et al., 2020), both of which are physiological factors linked with increased risk of sarcopenia (Nelke et al., 2019; Shou et al., 2020). Older adults are characterized by increased LPS levels that enhance toll-like receptor 4 (TLR4) signaling, promoting metabolic endotoxemia (Ghosh et al., 2015). Metabolic endotoxemia may induce systemic inflammation through reactive oxygen species production (Yuan et al., 2013) and activation of apoptotic pathways (i.e., nuclear factor $\kappa \mathrm{B}$, c-Jun $\mathrm{N}$-terminal kinase), downregulating immune function in older adults (Qian et al., 2012). Specifically, proinflammatory cytokines (i.e., IL-6 and TNF-a) may modulate LPS-induced proinflammatory responses through TLR4/Mal signaling pathway (Greenhill et al., 2011). Therefore, the altered function and composition of gut metabolites during aging may be responsible for metabolic perturbations that develop throughout lifespan.

Regarding gut physiology, amino acid absorption is modulated at the cellular level via active transport by the epithelial intestinal cells in the small intestine located at the surface of enterocytes (Mardinoglu et al., 2015). These transporters shuttle amino acids into the circulation (Broer, 2008) via the peptide transporter 1 carrier (Walther et al., 2019). Recent evidence implicates a role for the human microbial environment in amino acid homeostasis (Yatsunenko et al., 2012; Ticinesi et al., 2019). Specifically, human studies demonstrate that the microbial-derived amino acids, e.g., threonine and lysine, are incorporated into the free plasma amino acid pool following consumption of a moderate protein diet, suggesting 


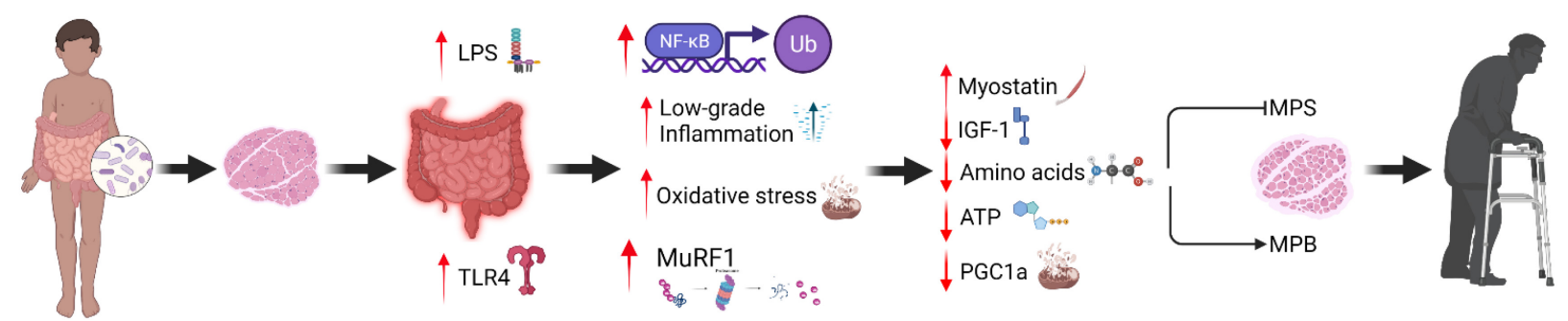

FIGURE 1 | Proposed mechanisms that underpin muscle anabolic resistance via changes in gut microbiota diversity.

that disruption of the gut microbiota environment may suppress the microbial-induced production of amino acids (Metges et al., 1999) and potentially lead to AR. Furthermore, during dietary protein restriction, the gut microbiota is reported to produce amino acids through de novo biosynthesis (Lin et al., 2017) and is implicated in amino acid homeostasis via FGF21 hepatic signaling (Martin et al., 2021). However, the role of the gut microbiome in modulating the uptake of amino acids, in particular the branched-chain amino acids, into the skeletal muscle cell is yet to be fully elucidated in humans. Future studies are warranted to fill this gap in knowledge, with the likely focus on leucine uptake by skeletal muscle given the role of leucine as both a substrate and signal for the stimulation of MPS (Anthony et al., 2001).

An alternative mechanism linking the gut microbiota with AR relates to insulin-like growth factor 1 (IGF-1). IGF-1 synthesis regulates nutrient sensing and the stimulation of MPS (Bian et al., 2020) that is modulated, in part, by the gut microbiota (Yan and Charles, 2018). This notion is supported by previous studies that associated circulating levels of IGF-1 with a decrease in Salmonella typhimurium and Burkholderia thailandensis infected mice compared to Escherichia coli O21: $\mathrm{H}+$ treated mice that retained IGF-1/Akt pathway capacity (Schieber et al., 2015). At the mechanistic level, IGF-1 is regulatory for muscle growth via the phosphatidylinositol 3kinase (PI3K)/Akt signaling pathway, and serves to suppress the mRNA transcription and translation process of MPS (Barclay et al., 2019). A recent study that utilized a $16 \mathrm{~S}$ ribosomal RNA gene sequencing approach revealed the gut microbiota of intestinal epithelial cell-specific IGF-1 knockout mice exhibited a disrupted intestinal homeostasis and epithelial regeneration in comparison to mice under normal pathological conditions (Zheng et al., 2018). Hence, it has been suggested that intestinal permeability due to microbiota dysbiosis may induce systemic inflammation (Soendergaard et al., 2017; Thevaranjan et al., 2017) and suppress IGF-1R sensitivity, thus initiating a catabolic response through MuRF-1 expression (Barclay et al., 2019). Given the potential physiological role of the gut microbiota in regulating skeletal muscle metabolism via the modulation of amino acid homeostasis and/or IGF-1 activity, a current focus of physiology research into healthy musculoskeletal aging relates to optimizing the gut microbiota (Ticinesi et al., 2019; Ni Lochlainn et al., 2021). It may be considered intuitive that lifestyle (i.e., physical activity, exercise, and diet) approaches targeted at modulating the microbial environment may mitigate AR associated with sarcopenia.

\section{MICROBIOME-CENTRIC DIETARY STRATEGIES TO COUNTER ANABOLIC RESISTANCE}

Microbial activity induced by indigestible amino acids promote the production of metabolic end products including short-chain fatty acids (SCFA; acetate, butyrate, propionate), branched-chain fatty acids (BCFA; valerate, isobutyrate, isovalerate), ammines, phenols, thiols, indoles, and ammonia. SCFA modulate epithelial cell function and microbiome physiology, serving as the primary energy source of colonocytes, and thus influence gastrointestinal health (Canfora et al., 2015). Specifically, acetate is utilized by skeletal muscle cells for ATP production, whereas the metabolic fate of butyrate and propionate primarily relates to gluconeogenesis and cholesterol synthesis (Byrne et al., 2015). SCFA are produced by dietary fiber fermentation (i.e., resistance starch, oligofructose, inulin, polydextrose, galactoolisaccharides) in the colon and are absorbed via the portal vein during lipid digestion (Chambers et al., 2018b). In addition, bacterial crossfeeding modulates SCFA production and substrate utilization with regards to human gut physiology (Ríos-Covián et al., 2016; Tsukuda and Yahagi, 2021). For instance, co-cultured Bacteroides uniformis and Escherichia coli were more effective in agarooligosaccharide degradation compared to their isolated properties (Li et al., 2014). Similarly, Bifidobacterium adolescentis co-cultured with Bifidobacterium infantis and Roseburia A2-183 strains exhibited a synergistic effect on agarotriose utilization and butyrate production (Belenguer et al., 2006; Li et al., 2014). Hence, cross-feeding of bacteria taxa is a primary contributor of SCFA synthesis and utilization that may provide useful insight in designing future microbiome-centric interventions to counter AR.

Several amino acids, including glycine, threonine, glutamate, lysine, arginine, ornithine, and aspartate, also play an important role in acetate production, whereas threonine, lysine, and glutamate are involved in butyrate synthesis, and threonine is involved in propionate synthesis (Davila et al., 2013). SCFA are increasingly recognized as modulators of skeletal muscle metabolism via action of the $G$ protein-coupled receptors GRP41 (FFAR3) and GRP43 (FFAR2) (Nilsson et al., 2003; 


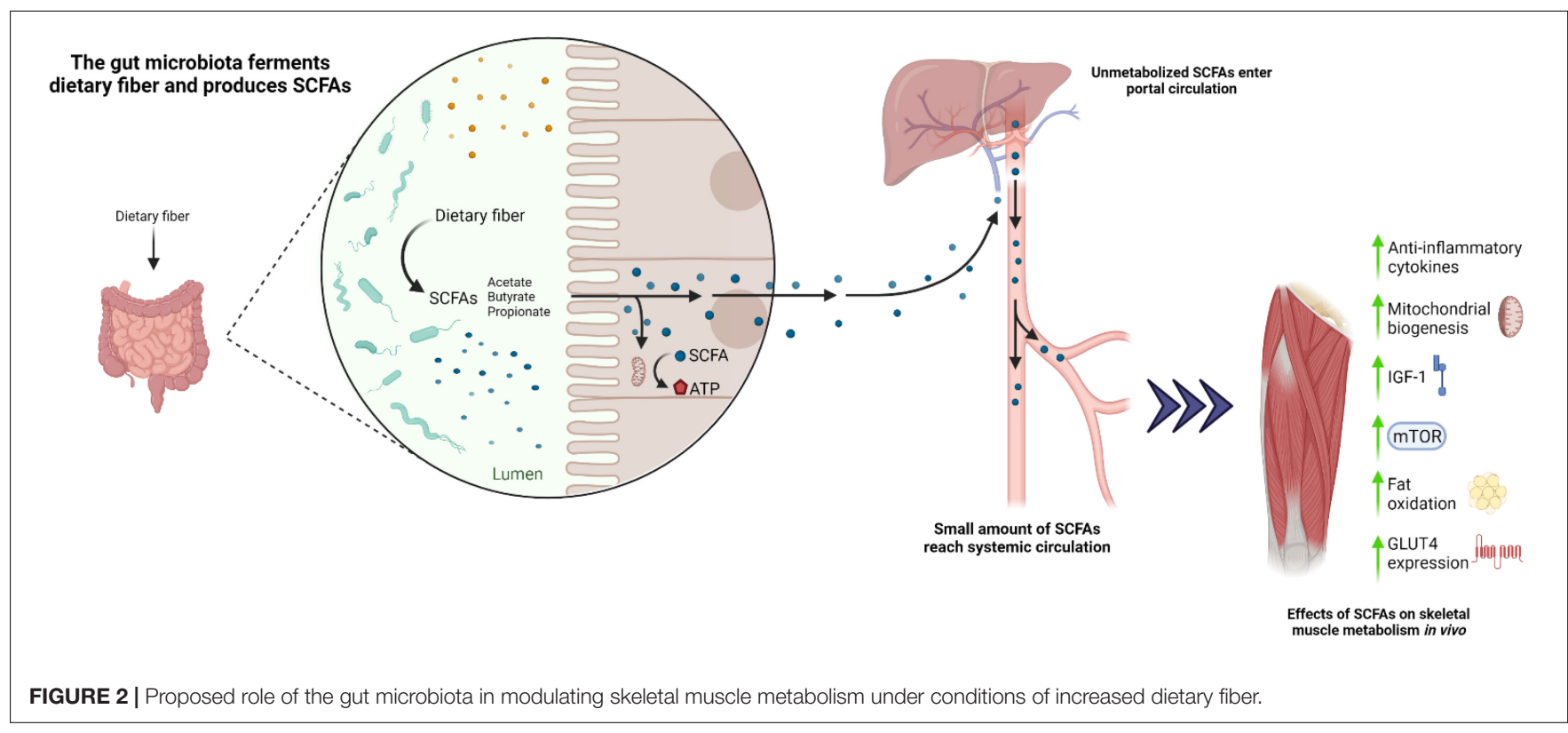

Kimura et al., 2014). GPR41 and GRP43 are understood to stimulate GLP-1 and PYY secretion and increase insulinmediated glucose uptake in skeletal muscle (Canfora et al., 2015). In vivo studies have demonstrated improvements in insulin sensitivity, mitochondrial biogenesis and function, reduced adiposity, and an increase in type I muscle fiber composition following SCFA administration (Fushimi et al., 2001; Gao et al., 2009; Hong et al., 2016; Zhou et al., 2021) that correspond with an increased myoglobin expression (Yamashita et al., 2014; Maruta et al., 2016; Figure 2). Accordingly, germfree mice supplemented with SCFA were shown to exhibit greater muscle (gastrocnemius) mass and strength compared to germ-free controls (Lahiri et al., 2019). Likewise, a 10week butyrate-enriched diet improved mitochondrial biogenesis, insulin sensitivity, and muscle (quadriceps and gastrocnemius) mass in aged mice compared to butyrate-free controls, whereas no distinguishable differences were observed between younger groups (Walsh et al., 2015). Collectively, these data imply that SCFA administration may be beneficial in mitigating AR in mice. Likewise, in a human study of young and older adults, the administration of butyrate and propionate was shown to improve fat oxidation, insulin sensitivity, and inflammatory profiles (Chambers et al., 2015, 2018a, 2019; Cleophas et al., 2019). Furthermore, an increased capacity for gut microbial synthesis of butyrate was associated with elevated Faecalibacterium prausnitzii and Butyricimonas virosa, and higher skeletal muscle index ( $\mathrm{Lv}$ et al., 2021). Interestingly, older adults with a higher dietary fiber density (grams of fiber consumed/100 kcal) displayed a positive relationship with increased whole body lean mass and butyrate-producing bacteria, including Ruminococcus, Lachnospira, and Clostridia (Barger et al., 2020)] compared with older adults consuming lower fiber intake. Consistent with this observation, 13 weeks of soluble fiber supplementation in older adults led to improvements in handgrip strength, although changes in gut microbiota and SCFA were not examined in this study (Buigues et al., 2016). These observations are in line with a recent study, highlighting an association of higher dietary fiber intake with increased skeletal muscle mass and strength in middle-aged to older adults (Frampton et al., 2021). To date, no clinical studies have investigated the direct impact of SCFA and high soluble fiber diets on skeletal muscle protein metabolism. Hence, future studies are warranted to investigate the chronic impact of manipulating dietary fiber content in promoting gut derived SCFA and modulating skeletal muscle metabolism and inflammation in older adults.

\section{PREBIOTIC AND PROBIOTIC SUPPLEMENTATION AS A STRATEGY TO COUNTER ANABOLIC RESISTANCE}

Preliminary evidence, based on a limited number of hypothesisdriven studies, suggests that probiotic supplementation may confer physiological benefits to host physiology bacteria and thus promote skeletal muscle anabolism. Several studies have investigated the impact of Lactobacilli administration on metabolic function. Specifically, there is evidence that Lactobacillus plantarum may exert muscle anabolic effects by enhancing protein assimilation and upregulating the activation of mTOR as a molecular driver of MPS, as demonstrated using Drosophila models (Storelli et al., 2011; Erkosar et al., 2015). This observation may be explained, at least in part, by a shift in gut microbiota to a higher abundance of butyrate-producing species, leading to an increased IGF-1 activity and reduced proinflammatory cytokine secretion, as observed in Lactobacillus supplemented mice compared to germ-free counterparts (Bindels et al., 2012; Chen et al., 2016, 2020). Furthermore, administration of Lactobacillus paracasei PS23 resulted in greater mitochondrial function and reduced inflammatory cytokine activity in senescence-accelerated mice, with potential 
implications for reducing sarcopenia risk (Chen et al., 2019). Consistent with this finding, the administration of probiotics containing Lactobacilli species was shown to reduce systemic levels of IL-6 and TNF-a (Barreto et al., 2014; Borzabadi et al., 2018), and improve amino acid absorption kinetics in humans (Jäger et al., 2020). Hence, there is physiological rationale, albeit relatively limited experiential evidence, to suggest that multiple Lactobacillus strains may mitigate AR through a concomitant decrease in systemic inflammation and a greater amino acid utilization in the gut.

The administration of multiple bacterial species may be another strategy to target the gut microbiota and counteract AR. Microbiota transplantation from healthy or undernourished infants into young germ-free mice has demonstrated an increased accumulation of Ruminococcus gnavus and Clostridium symbiosum that ameliorated lean body mass gains and muscle growth (Blanton et al., 2016). Consistent with this observation, the transfer of gut microbiota from lean vs. obese pigs to germfree coincided with marked increases in gastrocnemius muscle fiber size (Yan et al., 2016), thus highlighting the potential impact of administering multiple bacteria through nutritional targets (i.e., synbiotic supplementation).

The combination of prebiotics (non-digestible fiber) and probiotics, collectively termed synbiotics, provides an emerging nutritional strategy to ingest non-digestible fiber in order to promote the development of specific gut microbiota species. Synbiotics consist of Bifidobacterium and Lactobacillus species and have been shown to reduce lipid accumulation, enhance muscle performance, and improve gut barrier function in aged mice (Ni et al., 2019). Moreover, synbiotics may suppress lowgrade inflammation through SCFA administration, mediated via a greater composition of colonic bacteria communities in older adults (Lahtinen et al., 2009; Macfarlane et al., 2013), although this observation is not universal (Neto et al., 2013). Accordingly, probiotic (i.e., Lactobacillus salivarius, Lactobacillus plantarum TWK10) and prebiotic (i.e., inulin) supplementation has been proposed as a promising strategy to facilitate the provision of a healthy gut microbiota, reducing systemic inflammation and improving exercise performance and muscle strength (Xiao et al., 2014; Chen et al., 2019; Lee et al., 2020). Consistent with this notion, several experimental trials have demonstrated microbial enrichment accompanied by reduced proinflammatory cytokine secretion (Vulevic et al., 2008), improved insulin sensitivity (Cani et al., 2009; van der Beek et al., 2018), handgrip strength (Buigues et al., 2016) and frailty conditions (Theou et al., 2019), and increased SCFA concentrations (Rebello et al., 2016) in both young and older adults. Hence, various parameters (i.e., low-grade inflammation and insulin resistance) associated with AR may be attenuated with prebiotic, probiotic, and/or synbiotic administration. To our knowledge, no studies have investigated the impact of microbial species on the stimulation of MPS and/or activation of mTOR related signaling in older adults. Therefore, future studies are warranted to investigate the impact of prebiotic or probiotic supplementation on the production of gut microbiota strains and subsequent stimulation and/or suppression of MPS and MPB, respectively, in young and older adults.

\section{EXERCISE AS A STRATEGY TO COUNTER ANABOLIC RESISTANCE VIA THE MODULATION OF GUT MICROBIOTA}

Exercise/physical activity is a crucial component of any strategy designed to prevent and/or treat sarcopenia, which may be mediated, in part, by modulating the gut microbiome (Strasser et al., 2021). Accordingly, the impact of exercise training in modulating the gut microbiota has primarily been established in physically active populations and animal models in the context of aerobic-based exercise (Cerdá et al., 2016; Taniguchi et al., 2018; Morita et al., 2019; Przewłócka et al., 2020; Clauss et al., 2021). Specifically, Bacteroides fragilis gnotobiotic mice improved swimming exercise capacity and reduced physical fatigue compared to germ-free mice (Hsu et al., 2015), and similar results were observed in gnotobiotic models containing Eubacterium rectale, Lactobacillus plantarum, and Clostridium coccoides bacteria (Huang et al., 2019). Similarly, inoculation of Veillonella atypica in mice improved running exercise capacity and appeared to be mediated via the conversion of exercise-induced lactate to propionate (Scheiman et al., 2019), whereas the combination of aerobic exercise with Bifidobacterium longum administration may further improve aerobic capacity and inflammatory status as demonstrated in mice supplemented with a probiotic strain isolated from an elite Olympic athlete (Huang et al., 2020). Recently, Valentino et al. (2021) showed that antibiotic-treated mice resulted in a disrupted gut microbiome, which was correlated with less hypertrophy of soleus type I and IIa, and plantaris type IIb muscle fibers compared to untreated counterparts following progressive weighted wheel running (Valentino et al., 2021). Furthermore, another study characterized professional athletes as exhibiting more diverse microbial communities and bacterial species involved in SCFA production compared to age-matched sedentary individuals (Barton et al., 2018). This observation may explain the role of exercise training in promoting SCFA biosynthesis (Clarke et al., 2014; Dumas et al., 2017; Murtaza et al., 2019). Consistent with this observation, the implementation of a training program that combined aerobic and resistance exercise resulted in an increased abundance of Blautia, Dialister, and Roseburia, and decreased abundance of Proteobacteria and Gammaproteobacteria phylum in obese children (Quiroga et al., 2020). However, 12 weeks into the intervention, no differences in gut microbiota profile were detected between the obese children and an age-matched, healthy control cohort.

A bidirectional relationship between exercise and bacterial strains has been reported in older adults supplemented with Lactobacillus casei, which was associated with increased physical activity levels as measured via daily step count (Aoyagi et al., 2019). Observational data reveal that physically active older adults are characterized by an increased abundance of Bifidobacteriales and Clostridiales species (Castro-Mejía et al., 2020). This observation is not consistent with previous studies that indicated a decreased microbial diversity during sedentarism (Bressa et al., 2017; Castellanos et al., 2020). Moreover, 
differences in bacteria taxa have been observed through higher Faecalibacterium prausnitzii and lower Parasutterella excrementihominis between physically active and communitydwelling older adults (Fart et al., 2020). However, limited data in humans has been generated regarding the modulation of gut microbiota with resistance training in older adults.

Although endurance exercise confers multiple metabolic health benefits, including improved insulin sensitivity, mitochondrial function, and maximal oxygen consumption (Bird and Hawley, 2017; Hargreaves and Spriet, 2020), resistance training provides the most robust anabolic stimulus to mitigate age-related AR (Mcleod et al., 2019). Preliminary in vivo data suggest an improvement in gut microbiota diversity and composition in response to resistance training (Chen et al., 2021). Specifically, a previous study revealed a reduced relative abundance of pro-inflammatory-induced species, including Pseudomonas, Serratia, Comamonas, and Firmicutes/Bacteroidetes ratio that translated to a decrease in intestinal mucosal permeability and enriched SCFA-producing gut microbiota. Whereas no changes in the gut microbiome with resistance training were observed in young adults (Bycura et al., 2021), this study may be considered to lack statistical power in terms of microbiome sampling, and the short duration of intervention (8 week) implemented may have been insufficient to elicit detectable changes in the gut microbiome (Bycura et al., 2021). Interestingly, a seminal study by Fielding et al. (2019) revealed that transferring fecal samples from high-functioning older adults to mice increased the abundance of Barnesiella intestinihominis bacteria that corresponded with improved grip strength compared with lowfunctioning-colonized mice (Fielding et al., 2019). Indeed, the high-functioning-colonized mice displayed a higher number of Prevotellaceae family, Prevotella and Barnesiella genus, and Barnesiella intestinihominis species compared to lowfunctioning-colonized mice. These findings align with previous work linking an elevated Prevotellaceae family profile to young professional athletes (Clarke et al., 2014), and Prevotella and Barnesiella to less frail phenotypes (Van Tongeren et al., 2005; Claesson et al., 2012; Verdi et al., 2018). Accordingly, these data suggest that these microbes are associated with better physical conditioning. Moreover, genes derived from Barnesiella and Prevotellaceae may produce SCFAs, that could further explain the improved muscle functional outcomes germ-free mice following a SCFA cocktail (Lahiri et al., 2019). Taken

\section{REFERENCES}

Alhmoud, T., Kumar, A., Lo, C., Al-Sadi, R., Clegg, S., Alomari, I., et al. (2019). Investigating intestinal permeability and gut microbiota roles in acute coronary syndrome patients. Hum. Microbiome J. 13:100059. doi: 10.1016/j.humic.2019. 100059

Anthony, J. C., Anthony, T. G., Kimball, S. R., Jefferson, L. S., et al. (2001). Signaling pathways involved in translational control of protein synthesis in skeletal muscle by leucine. J. Nutr. 131, 856S-860S.

Aoyagi, Y., Amamoto, R., Park, S., and Honda, Y. (2019). Independent and interactive effects of habitually ingesting fermented milk products containing lactobacillus casei strain shirota and of engaging in moderate habitual daily together, these preliminary findings indicate that resistance exercise may lead to the production of SCFA metabolites, contributing to improved muscle strength, although this thesis warrants direct investigation in older adults. Future studies designed to examine the impact of resistance training on bacteria taxa and SCFA production and their influence on the gut microbiome in young and older adults would provide more reliable conclusions in humans.

\section{CONCLUSION}

Preliminary evidence, based on a limited number of hypothesisdriven studies primarily conducted using animal models, suggests a mechanistic action for the gut microbiota in countering age-related AR and sarcopenia risk. However, at present, clinical trials are warranted to validate these microbialinduced outcomes on skeletal muscle using in vivo human models. Based on findings from animal- and cell-based models, there is evidence to suggest that improvements in de novo amino acid biosynthesis may correspond with the maintenance of gut microbiota diversity through promotion of SCFA via dietary fiber and protein consumption. Moreover, in vivo experiments have demonstrated an increased SCFA production following exercise that may attenuate AR by enhancing amino acid utilization and reducing levels of low-grade inflammation. Moving forward, the design of lifestyle approaches that combine increased dietary fiber and protein intake, probiotic supplementation and resistance training may be effective in optimizing gut microbiota composition with implications for muscle health in older adults at risk of sarcopenia.

\section{AUTHOR CONTRIBUTIONS}

$\mathrm{KP}$ and $\mathrm{OW}$ conceived and wrote the initial draft of the manuscript. EC and $\mathrm{MN}$ reviewed and revised the manuscript. All authors contributed to the article and approved the submitted version.

\section{ACKNOWLEDGMENTS}

Figures were created with BioRender.com.

physical activity on the intestinal health of older people. Front. Microbiol. 10:1477. doi: 10.3389/fmicb.2019.01477

Azzolino, D., Passarelli, P. C., De Angelis, P., Piccirillo, G. B., D’addona, A., Cesari, M., et al. (2019). Poor oral health as a determinant of malnutrition and sarcopenia. Nutrients 11:2898. doi: 10.3390/nu11122898

Badal, V. D., Vaccariello, Murray, E. R., Yu, K. E., Knight, R., Jeste, D. V., et al. (2020). The gut microbiome, aging, and longevity: a systematic review. Nutrients 12:3759. doi: 10.3390/nu12123759

Barclay, R. D., Burd, N. A., Tyler, C., Tillin, N. A., Mackenzie, R. W., et al. (2019). The role of the IGF-1 signaling cascade in muscle protein synthesis and anabolic resistance in aging skeletal muscle. Front. Nutr. 6:146. doi: 10.3389/fnut.2019. 00146 
Barger, K., Langsetmo, L., Orwoll, E. S., and Lustgarten, M. S. (2020). Investigation of the diet-gut-muscle axis in the osteoporotic fractures in men study. J. Nutr. Heal. Aging. 24, 445-452. doi: 10.1007/s12603-020-1344-1

Barreto, F. M., Simao, A. N. C., Morimoto, H. K., Lozovoy, M. A. B., Dichi, I., da Miglioranza, L. H. S., et al. (2014). Beneficial effects of Lactobacillus plantarum on glycemia and homocysteine levels in postmenopausal women with metabolic syndrome. Nutrition 30, 939-942. doi: 10.1016/j.nut.2013.12.004

Barton, W., Penney, N. C., Cronin, O., Garcia-perez, I., Molloy, M. G., Holmes, E., et al. (2018). The microbiome of professional athletes differs from that of more sedentary subjects in composition and particularly at the functional metabolic level. Gut 67, 625-633.

Belenguer, A., Duncan, S. H., Calder, A. G., Holtrop, G., Louis, P., Lobley, G. E., et al. (2006). Two routes of metabolic cross-feeding between bifidobacterium adolescentis and butyrate-producing anaerobes from the human gut. Appl. Environ. Microbiol. 72, 3593-3599. doi: 10.1128/aem.72.5.3593-3599.2006

Belkaid, Y., and Hand, T. (2014). Role of the microbiota in immunity and inflammation. Cell 157, 121-141. doi: 10.1016/j.cell.2014.03.011

Beyer, I., Mets, T., and Bautmans, I. (2012). Chronic low-grade inflammation and age-related sarcopenia. Curr. Opin. Clin. Nutr. Metab. Care 15, 12-22. doi: 10.1097/mco.0b013e32834dd297

Biagi, E., Nylund, L., Candela, M., Ostan, R., Bucci, L., Pini, E., et al. (2010). Through ageing, and beyond: gut microbiota and inflammatory status in seniors and centenarians. PLoS One 5:e10667. doi: 10.1371/journal.pone.0010667

Bian, A., Ma, Y., Zhou, X., Guo, Y., Wang, W., Zhang, Y., et al. (2020). Association between sarcopenia and levels of growth hormone and insulin-like growth factor-1 in the elderly. BMC Musculoskelet. Disord. 21:214. doi: 10.1186/s12891020-03236-y

Bindels, L. B., Beck, R., Schakman, O., Martin, J. C., de Backer, F., Sohet, F. M., et al. (2012). Restoring specific lactobacilli levels decreases inflammation and muscle atrophy markers in an acute leukemia mouse model. PLoS One 7:e37971. doi: 10.1371/journal.pone.0037971

Biolo, G., Zhang, X.-J., and Wolfe, R. R. (1995). Role of membrane transport in interorgan amino acid flow between muscle and small intestine. Metabolism 44, 719-724. doi: 10.1016/0026-0495(95)90183-3

Bird, S. R., and Hawley, J. A. (2017). Update on the effects of physical activity on insulin sensitivity in humans. BMJ Open Sport Exerc. Med. 2:e000143. doi: 10.1136/bmjsem-2016-000143

Blanton, L. V., Charbonneau, M. R., Salih, T., Barratt, M. J., Venkatesh, S., Ilkaveya, O., et al. (2016). Gut bacteria that rescue growth impairments transmitted by immature microbiota from undernourished children. Science 351:10. doi: 10.1126/science.aad3311

Bohé, J., Low, A., Wolfe, R. R., and Rennie, M. J. (2003). Human muscle protein synthesis is modulated by extracellular, not intramuscular amino acid availability: a dose-response study. J. Physiol. 552, 315-324. doi: 10.1113/ jphysiol.2003.050674

Boirie, Y., Gachon, P., and Beaufrère, B. (1997). Splanchnic and whole-body leucine kinetics in young and elderly men. Am. J. Clin. Nutr. 65, 489-495. doi: 10.1093/ ajcn/65.2.489

Borzabadi, S., Oryan, S., Eidi, A., Aghadavod, E., and Kakhaki, R. D. (2018). The effects of probiotic supplementation on gene expression related to inflammation, insulin and lipid in patients with Parkinson's disease: a randomized, double-blind, placebo-controlled trial. Arch. Iran Med. 21, 289295.

Bosco, N., and Noti, M. (2021). The aging gut microbiome and its impact on host immunity. Genes Immun. 23, 289-303. doi: 10.1038/s41435-021-00126-8

Bressa, C., Bailen-Andrino, M., Perez-Santiago, J., Gonzaez-Soltero, R., Perez, M., Montalvo-Lominchar, M. G., et al. (2017). Differences in gut microbiota profile between women with active lifestyle and sedentary women. PLoS One 12:e0171352. doi: 10.1371/journal.pone.0171352

Broer, S. (2008). Amino acid transport across mammalian intestinal and renal epithelia. Physiol. Rev. 88, 249-286. doi: 10.1152/physrev.00018.2006

Buigues, C., Fernández-Garrido, J., Pruimboom, L., Hoogland, A. J., NavarroMartínez, R., Martínez-Martínez, M., et al. (2016). Effect of a prebiotic formulation on frailty syndrome: a randomized, double-blind clinical trial. Int. J. Mol. Sci. 17:932. doi: 10.3390/ijms17060932

Burd, N. A., Gorissen, S. H., and Van Loon, L. J. C. (2013). Anabolic resistance of muscle protein synthesis with aging. Exerc. Sport Sci. Rev. 41, 169-173. doi: $10.1097 /$ jes.0b013e318292f3d5
Burd, N. A., Yang, Y., Moore, D. R., Tang, J. E., Tarnopolsky, M. A., Phillips, S. M., et al. (2012). Greater stimulation of myofibrillar protein synthesis with ingestion of whey protein isolate vs. micellar casein at rest and after resistance exercise in elderly men. Br. J. Nutr. 108, 958-962. doi: 10.1017/s0007114511006271

Bycura, D., Santos, A. C., Shiffer, A., Kyman, S., Winfree, K., Sutliffe, J., et al. (2021). Impact of different exercise modalities on the human gut microbiome. Sports 9:14. doi: 10.3390/sports9020014

Byrne, C. S., Chambers, E. S., Morrison, D. J., and Frost, G. (2015). The role of short chain fatty acids in appetite regulation and energy homeostasis. Int. J. Obes. 39, 1331-1338. doi: 10.1038/ijo.2015.84

Canfora, E. E., Jocken, J. W., and Blaak, E. E. (2015). Short-chain fatty acids in control of body weight and insulin sensitivity. Nat. Rev. Endocrinol. 11, 577-591. doi: 10.1038/nrendo.2015.128

Cani, P. D., Lecourt, E., Dewulf, E. M., Sohet, F. M., Pachikian, B. D., Naslain, D., et al. (2009). Gut microbiota fermentation of prebiotics increases satietogenic and incretin gut peptide production with consequences for appetite sensation and glucose response after a meal. Am. Soc. Nutr. 90, 1236-1243. doi: 10.3945/ ajcn.2009.28095

Cao, L., and Morley, J. E. (2016). Sarcopenia is recognized as an independent condition by an international classification of disease, tenth revision, clinical modification (ICD-10-CM) code. J. Am. Med. Dir. Assoc. 17, 675-677. doi: 10.1016/j.jamda.2016.06.001

Castellanos, N., Diez, G. G., Antúnez-almagro, C., Bailén, M., Waite, D. W., Barton, W., et al. (2020). A critical mutualism - competition interplay underlies the loss of microbial diversity in sedentary lifestyle. Front. Microbiol. 10:3142. doi: 10.3389/fmicb.2019.03142

Castro-Mejía, J. L., Bekzod, K., Krych, L., Bulow, J., Bechshøft, R. L., Højfeldt, G., et al. (2020). Physical fitness in community-dwelling older adults is linked to dietary intake, gut microbiota, and metabolomic signatures. Aging Cell 19:e13105.

Cerdá, B., Pérez, M., Pérez-Santiago, Jennifer, D., Tornero-Aguiler, J. F., GonzálezSoltero, R., et al. (2016). Gut microbiota modification: another piece in the puzzle of the benefits of physical exercise in health? Front. Physiol. 7:51. doi: 10.3389/fphys.2016.00051

Chambers, E. S., Byrne, C. S., Aspey, K., Chen, Y., Saadiyah, M., Douglas, K., et al. (2018a). Acute oral sodium propionate supplementation raises resting energy expenditure and lipid oxidation in fasted humans. Diab. Obes Metab Obes 20, 1034-1039. doi: 10.1111/dom.13159

Chambers, E. S., Preston, T., Frost, G., Morrison, D. J., et al. (2018b). Role of gut microbiota-generated short-chain fatty acids in metabolic and cardiovascular health. Curr. Nutr. Rep. 7, 198-206. doi: 10.1007/s13668-018-0248-8

Chambers, E. S., Byrne, C. S., Morrison, D. J., Murphy, K. G., Preston, T., Tedford, C., et al. (2019). Dietary supplementation with inulin-propionate ester or inulin improves insulin sensitivity in adults with overweight and obesity with distinct effects on the gut microbiota, plasma metabolome and systemic inflammatory responses: a randomised cross-over trial. Gut 68, 1430-1438. doi: 10.1136/ gutjnl-2019-318424

Chambers, E. S., Viardot, A., Psichas, A., Morrison, D. J., Murphy, K. G., Zacvarghese, S. E. K., et al. (2015). Effects of targeted delivery of propionate to the human colon on appetite regulation, body weight maintenance and adiposity in overweight adults. Gut 64, 1744-1754. doi: 10.1136/gutjnl-2014-307913

Chen, H., Shen, L., Liu, Y., Ma, X., Long, L., Ma, X., et al. (2021). Strength exercise confers protection in central nervous system autoimmunity by altering the gut microbiota. Front. Immunol. 12:628629. doi: 10.3389/fimmu.2021.628629

Chen, L. H., Huang, S. Y., Huang, K. C., Hsu, C. C., Yang, K. C., Ai, L., et al. (2019). Lactobacillus paracasei PS23 decelerated age - related muscle loss by ensuring mitochondrial function in SAMP8 mice. Aging 11, 756-770. doi: 10.18632/aging. 101782

Chen, Y., Liao, C., Huang, Y.-C., Chen, M.-Y., Huang, C.-C., Chen, W.C., et al. (2020). Proteome and microbiota analysis highlight Lactobacillus plantarum TWK10 supplementation improves energy metabolism and exercise performance in mice. Food Sci. Nutr. 8, 3525-3534. doi: 10.1002/fsn3.1635

Chen, Y. M., Wei, L., Chiu, Y. S., Hsu, Y. J., Tsai, T. Y., Wang, M. F., et al. (2016). Lactobacillus plantarum TWK10 supplementation improves exercise performance and increases muscle mass in mice. Nutrients 8:205. doi: 10.3390/ nu8040205

Choi, Y., Kwon, Y., Kim, D., Jeon, J., Jang, S. C., Wang, T., et al. (2015). Gut microbe-derived extracellular vesicles induce insulin resistance, thereby 
impairing glucose metabolism in skeletal muscle. Sci. Rep. 5:15878. doi: 10. 1038/srep15878

Claesson, M. J., Jeffery, I. B., Conde, S., Power, S. E., O'connor, E. M., Cusack, S., et al. (2012). Gut microbiota composition correlates with diet and health in the elderly. Nature 488, 178-184. doi: 10.1038/nature11319

Clarke, S. F., Murphy, E. F., Sullivan, O. O., Lucey, A. J., Humphreys, M., Hogan, A., et al. (2014). Exercise and associated dietary extremes impact on gut microbial diversity. Gut 63, 1913-1920. doi: 10.1136/gutjnl-2013-306541

Clauss, M., Gérard, P., Mosca, A., and Leclerc, M. (2021). Interplay between exercise and gut microbiome in the context of human health and performance. Front. Nutr. 8:637010. doi: 10.3389/fnut.2021.637010

Cleasby, M. E., Jamieson, P. M., and Atherton, P. J. (2016). Insulin resistance and sarcopenia: mechanistic links between common co-morbidities. J. Endocrinol. 229, R67-R81. doi: 10.1530/JOE-15-0533

Cleophas, M. C. P., Ratter, J. M., Bekkering, S., Quintin, J., Schraa, K., Stroes, E. S., et al. (2019). Effects of oral butyrate supplementation on inflammatory potential of circulating peripheral blood mononuclear cells in healthy and obese males. Sci. Rep. 9:775. doi: 10.1038/s41598-018-37246-7

Coman, V., and Cristian, D. (2020). Gut microbiota and old age: modulating factors and interventions for healthy longevity. Exp. Gerontol. 141:111095. doi: 10.1016/j.exger.2020.111095

Cruz-Jentoft, A. J., Bahat, G., Bauer, J., Boirie, Y., Bruyère, O., Cederholm, T., et al. (2019). Sarcopenia: revised European consensus on definition and diagnosis. Age Ageing 48, 16-31. doi: 10.1093/ageing/afy169

Cuthbertson, D., Smith, K., Babraj, J., Leese, G., Waddell, T., Atherton, P., et al. (2005). Anabolic signaling deficits underlie amino acid resistance of wasting, aging muscle. FASEB J. 19, 422-424.

Davila, A. M., Blachier, F., Gotteland, M., Andriamihaja, M., Benetti, P. H., Sanz, Y., et al. (2013). Intestinal luminal nitrogen metabolism: role of the gut microbiota and consequences for the host. Pharmacol. Res. 68, 95-107. doi: 10.1016/j.phrs. 2012.11.005

Dumas, M. E., Rothwell, A. R., Hoyles, L., Aranias, T., Chilloux, J., Calderari, S., et al. (2017). Microbial-Host co-metabolites are prodromal markers predicting phenotypic heterogeneity in behavior, obesity, and impaired glucose tolerance. Cell Rep. 20, 136-148. doi: 10.1016/j.celrep.2017.06.039

Durham, W. J., Casperson, S. L., Dillon, E. L., Keske, M. A., Paddon-jones, D., Sanford, A. P., et al. (2010). Age related anabolic resistance after endurance-type exercise in healthy humans. FASEB J. 24, 4117-4127. doi: 10.1096/fj.09-150177

Erkosar, B., Storelli, G., Mitchell, M., Bozonnet, L., Bozonnet, N., Leulier, F., et al. (2015). Pathogen virulence impedes mutualist-mediated enhancement of host juvenile growth via inhibition of protein digestion article pathogen virulence impedes mutualist - mediated enhancement of host juvenile growth via inhibition of protein digestion. Cell Host Microbe 18, 445-455. doi: 10.1016/ j.chom.2015.09.001

Fart, F., Rajan, S. K., Wall, R., Rangel, I., Ganda-Mall, J. P., Tingö, L., et al. (2020). Differences in gut microbiome composition between senior orienteering athletes and community-dwelling older adults. Nutrients 12:2610. doi: 10.3390/ nu12092610

Fielding, R. A., Reeves, A. A., Jasuja, R., Liu, C., Barrett, B. B., Lustgarten, M. S., et al. (2019). Muscle strength is increased in mice that are colonized with microbiota from high-functioning older adults. Exp. Gerontol. 127:110722. doi: 10.1016/j.exger.2019.110722

Frampton, J., Murphy, K. G., Frost, G., and Chambers, E. S. (2021). Higher dietary fibre intake is associated with increased skeletal muscle mass and strength in adults aged 40 years and older. J. Cachexia Sarcopenia Muscle. Online ahead of print.

Frampton, J., Murphy, K. G., Frost, G., Chambers, E. S., et al. (2020). Short-chain fatty acids as potential regulators of skeletal muscle metabolism and function. Nat. Metab. 2, 840-848. doi: 10.1038/s42255-020-0188-7

Fushimi, T., Tayama, K., Fukaya, M., Kitakoshi, K., Nakai, N., Tsukamoto, Y., et al. (2001). Acetic acid feeding enhances glycogen repletion in liver and skeletal muscle of rats. J. Nutr. 131, 1973-1977. doi: 10.1093/jn/131.7.1973

Gao, Z., Yin, J., Zhang, J., Ward, R. E., Martin, R. J., Lefevre, M., et al. (2009). Butyrate improves insulin sensitivity and increases energy expenditure in mice. Diab. Metab. Res. Rev. 58, 1509-1517. doi: 10.2337/db08-1637

Ghosh, S., Lertwattanarak, R., Garduño, J. D. J., Galeana, J. J., Li, J., Zamarripa, F., et al. (2015). Elevated muscle TLR4 expression and metabolic endotoxemia in human aging. J. Gerontol. Med. Sci. 70, 232-246. doi: 10.1093/gerona/glu067
Greenhill, C. J., Rose-john, S., Ferlin, W., Neill, L. O., Hertzog, P., Mansell, A., et al. (2011). IL-6 trans-signaling modulates TLR4-Dependent inflammatory responses via STAT3. J. Immunol. 186, 1199-1208. doi: 10.4049/jimmunol. 1002971

Grosicki, G. J., Fielding, R. A., and Lustgarten, M. S. (2018). Gut microbiota contribute to age-related changes in skeletal muscle size, composition, and function: biological basis for a gut-muscle axis. Calcif. Tissue Int. 102, 433-442. doi: 10.1007/s00223-017-0345-5

Guillet, C., Prod, M., Balage, M., Gachon, P., Giraudet, C., Morin, L., et al. (2004). Impaired anabolic response of muscle protein synthesis is associated with S6K1 dysregulation in elderly humans. FASEB J. 16, 1586-1587. doi: 10.1096/fj.03$1341 \mathrm{fje}$

Guinane, C. M., and Cotter, P. D. (2013). Role of the gut microbiota in health and chronic gastrointestinal disease: understanding a hidden metabolic organ. Therap. Adv. Gastroenterol. 6, 295-308. doi: 10.1177/1756283x134 82996

Hargreaves, M., and Spriet, L. L. (2020). Skeletal muscle energy metabolism during exercise. Nat. Metab. 2, 817-828. doi: 10.1038/s42255-020-0251-4

Hong, J., Jia, Y., Pan, S., Jia, L., Li, H., Han, Z., et al. (2016). Butyrate alleviates high fat diet-induced obesity through activation of adiponectin-mediated pathway and stimulation of mitochondrial function in the skeletal muscle of mice. Oncotarget 7, 56071-56082. doi: 10.18632/oncotarget.11267

Hsu, Y. J., Chiu, C. C., Li, Y. P., Huang, W. C., Huang, Y., Te, et al. (2015). Effect of intestinal microbiota on exercise performance in mice. J. Strength Cond. Res. 29, 552-558. doi: $10.1519 /$ jsc. 0000000000000644

Huang, W.-C., Chen, Y.-H., Chuang, H.-L., Chiu, C.-C., Lin, L., et al. (2019). Investigation of the effects of microbiota on exercise physiological adaption, performance, and energy utilization using a gnotobiotic animal model. Front. Microbiol. 10:1906. doi: 10.3389/fmicb.2019.01906

Huang, W.-C., Hsu, Y.-J., Huang, C.-C., Liu, H.-C., and Lee, M.-C. (2020). Exercise training combined with bifidobacterium longum OLP-01 supplementation improves exercise physiological adaption and performance. Nutrients 12:1145. doi: $10.3390 /$ nu12041145

Jackson, M. J., and McArdle, A. (2016). Role of reactive oxygen species in agerelated neuromuscular deficits. J. Physiol. 8, 1979-1988. doi: 10.1113/jp270564

Jäger, R., Zaragoza, J., Purpura, M., Iametti, S., Marengo, M., Tinsley, G. M., et al. (2020). Probiotic administration increases amino acid absorption from plant protein: a placebo-controlled, randomized, double-blind, multicenter, crossover study. Probiot. Antimicrob Proteins 12, 1330-1339. doi: 10.1007/s12602-02009656-5

Kang, L., Li, P., Wang, D., Wang, T., Hao, D., Qu, X., et al. (2021). Alterations in intestinal microbiota diversity, composition, and function in patients with sarcopenia. Sci. Rep. 11:4628.

Kimball, S. R., and Jefferson, L. S. (2002). Control of protein synthesis by amino acid availability. Curr. Opin. Clin. Nutr. Metab. Care 5, 63-67. doi: 10.1097/ 00075197-200201000-00012

Kimura, I., Inoue, D., Hirano, K., and Tsujimoto, G. (2014). The SCFA receptor GPR43 and energy metabolism. Front. Endocrinol. 5:85. doi: 10.3389/fendo. 2014.00085

Kumar, V., Selby, A., Rankin, D., Patel, R., Atherton, P., Hildebrandt, W., et al. (2009). Age-related differences in the dose-response relationship of muscle protein synthesis to resistance exercise in young and old men. J. Physiol. 587, 211-217. doi: 10.1113/jphysiol.2008.164483

Lahiri, S., Kim, H., Garcia-Perez, I., Reza, M. M., Martin, K. A., Kundu, P., et al. (2019). The gut microbiota influences skeletal muscle mass and function in mice. Sci. Transl. Med. 11:eaan5662. doi: 10.1126/scitranslmed.aan5662

Lahtinen, S. J., Tammela, L., Ahokoski, H., Mykkänen, H., et al. (2009). Probiotics modulate the Bifidobacterium microbiota of elderly nursing home residents. Age 31, 59-66. doi: 10.1007/s11357-008-9081-0

Langille, M. G. I., Meehan, C. J., Koenig, J. E., Dhanani, A. S., Rose, R. A., Howlett, S. E., et al. (2014). Microbial shifts in the aging mouse gut. Microbiome 2:50.

Larsen, N., Vogensen, F. K., Van Den Berg, F. W. J., Nielsen, D. S., Andreasen, A. S., Pedersen, B. K., et al. (2010). Gut microbiota in human adults with type 2 diabetes differs from non-diabetic adults. PLoS One 5:e9085. doi: 10.1371/ journal.pone.0009085

Le Chatelier, E., Nielsen, T., Qin, J., Prifti, E., Hildebrand, F., Falony, G., et al. (2013). Richness of human gut microbiome correlates with metabolic markers. Nature 500, 541-546. 
Lee, M.-C., Hsu, Y.-J., Ho, H.-H., Hsieh, S.-H., Kuo, Y.-W., Sung, H.-C., et al. (2020). Lactobacillus salivarius Subspecies salicinius SA-03 is a new probiotic capable of enhancing exercise performance and decreasing fatigue. Microorganisms 8:545. doi: 10.3390/microorganisms8040545

Li, M., Li, G., Zhu, L., Yin, Y., Zhao, X., Xiang, C., et al. (2014). Isolation and characterization of an Agaro-Oligosaccharide (AO)-hydrolyzing bacterium from the gut microflora of Chinese individuals. PLoS One 9:e91106. doi: 10. 1371/journal.pone.0091106

Liang, H., Hussey, S. E., Sanchez-Avila, A., Tantiwong, P., and Musi, N. (2013). Effect of lipopolysaccharide on inflammation and insulin action in human muscle. PLoS One 8:e63983. doi: 10.1371/journal.pone.0063983

Lin, R., Liu, W., Piao, M., and Zhu, H. (2017). A review of the relationship between the gut microbiota and amino acid metabolism. Amino Acids. 49, 2083-2090. doi: 10.1007/s00726-017-2493-3

Ling, Z., Liu, X., Cheng, Y., Yan, X., and Wu, S. (2020). Gut microbiota and aging. Crit. Rev. Food Sci. Nutr. 90, 351-371.

Liu, C., Cheung, W., Li, J., Chow, S. K., Yu, J., Wong, S. H., et al. (2021). Understanding the gut microbiota and sarcopenia: a systematic review. J. Cachexia Sarcopenia Muscle. Online ahead of print.

Lluch, J., Servant, F., Pa, S., Valle, C., Vali, S., Kuchly, C., et al. (2015). The characterization of novel tissue microbiota using an optimized $16 \mathrm{~S}$ metagenomic sequencing pipeline. PLoS One 10:e0142334. doi: 10.1371/journal. pone. 0142334

Lustgarten, M. S. (2016). Classifying aging as a disease: the role of microbes. Front. Genet. 7:212. doi: 10.3389/fgene.2016.00212

Lv, W., Lin, X., Shen, H., Liu, H., Qiu, X., Li, B., et al. (2021). Human gut microbiome impacts skeletal muscle mass via gut microbial synthesis of the short-chain fatty acid butyrate among healthy menopausal women. J. Cachexia Sarcopenia Muscle. Online ahead of print.

Macfarlane, S., Cleary, S., Bahrami, B., Reynolds, N., Macfarlane, G. T., et al. (2013). Synbiotic consumption changes the metabolism and composition of the gut microbiota in older people and modifies inflammatory processes: a randomised, double-blind, placebo-controlled crossover study. Aliment. Pharmacol. Ther. 38, 804-816. doi: 10.1111/apt.12453

Maes, M., Kubera, M., Leunis, J.-C., and Berk, M. (2012). Increased IgA and IgM responses against gut commensals in chronic depression: further evidence for increased bacterial translocation or leaky gut. J. Affect. Disord. 141, 55-62. doi: 10.1016/j.jad.2012.02.023

Mahnic, A., Breskvar, M., Dzeroski, S., Skok, P., Pintar, S., Rupnik, M., et al. (2020). Distinct types of gut microbiota dysbiosis in hospitalized gastroenterological patients are disease non-related and characterized with the predominance of either Enterobacteriaceae or Enterococcus. Front. Microbiol. 11:120. doi: 10. 3389/fmicb.2020.00120

Manickam, R., Yun, H., Oh, P., Tan, C. K., and Paramalingam, E. (2018). Metronidazole causes skeletal muscle atrophy and modulates muscle chronometabolism. Int. J. Mol. Sci. 19:2418. doi: 10.3390/ijms19082418

Mardinoglu, A., Shoaie, S., Bergentall, M., Ghaffari, P., Zhang, C., Larsson, E., et al. (2015). The gut 434 microbiota modulates host amino acid and glutathione metabolism in mice. Mol. Syst. Biol. 11:834. doi: 10.15252/msb.20156487

Mariat, D., Firmesse, O., Levenez, F., Guimar, V. D., Sokol, H., Doré, J., et al. (2009). The Firmicutes/Bacteroidetes ratio of the human microbiota changes with age. BMC Microbiol. 9:123. doi: 10.1186/1471-2180-9-123

Martin, A., Ecklu-mensah, G., Gilbert, J., Devkota, S., Ha, C. W. Y., Hendrick, G., et al. (2021). Gut microbiota mediate the FGF21 adaptive stress response to chronic dietary protein-restriction in mice. Nat. Commun. 12:3838.

Martin, A. M., Sun, E. W., Rogers, G. B., Keating, D. J., et al. (2019). The influence of the gut microbiome on host metabolism through the regulation of gut hormone release. Front. Physiol. 10:428. doi: 10.3389/fphys.2019.00428

Maruta, H., Yoshimura, Y., Araki, A., Kimoto, M., Takahashi, Y., Yamashita, H., et al. (2016). Activation of AMP-activated protein kinase and stimulation of energy metabolism by acetic acid in L6 myotube cells. PLoS One 11:e0158055. doi: 10.1371/journal.pone.0158055

Mcleod, J. C., Stokes, T., Phillips, S. M., and Phillips, S. M. (2019). Resistance exercise training as a primary countermeasure to age-related Chronic disease. Front. Physiol. 10:645. doi: 10.3389/fphys.2019.00645

Metges, C. C., El-Khoury, A. E., Henneman, L., Petzke, K. J., Grant, I., Bedri, S., et al. (1999). Availability of intestinal microbial lysine for whole body lysine homeostasis in human subjects. Am. J. Physiol. Endocrinol. Metab. 277, E597-E607.

Milani, C., Ferrario, C., Turroni, F., Duranti, S., Mangifesta, M., Sinderen, D., et al. (2016). The human gut microbiota and its interactive connections to diet. J. Hum. Nutr. Diet. 29, 539-546. doi: 10.1111/jhn.12371

Moore, D. R., Churchward-Venne, T. A., Witard, O., Breen, L., Burd, N. A., Tipton, K. D., et al. (2015). Protein ingestion to stimulate myofibrillar protein synthesis requires greater relative protein intakes in healthy older versus younger men. J. Gerontol - Ser A Biol. Sci. Med. Sci. 70, 57-62. doi: 10.1093/gerona/ glu103

Morita, E., Yokoyama, H., Imai, D., Takeda, R., Ota, A., Kawai, E., et al. (2019). Aerobic exercise training with brisk walking increases intestinal Bacteroides in healthy elderly women. Nutrients 11:868. doi: 10.3390/nu11040868

Moszak, M., Szulińska, M., and Bogdański, P. (2020). You are what you eatthe relationship between diet, microbiota, and metabolic disorders - a review. Nutrients 12:1096. doi: 10.3390/nu12041096

Murtaza, N., Burke, L. M., Vlahovich, N., Charlesson, B., O’Neill, H., Ross, M. L., et al. (2019). The effects of dietary pattern during intensified training on stool microbiota of elite race walkers. Nutrients 11:261. doi: 10.3390/nu11020261

Nagpal, R., Mainali, R., Ahmadi, S., Wang, S., Singh, R., et al. (2018). Gut microbiome and aging: physiological and mechanistic insights. Nutr. Healthy Ageing 4, 267-285. doi: 10.3233/nha- 170030

Nay, K., Jollet, M., Goustard, B., Baati, N., Vernus, B., Pontones, M., et al. (2019). Gut bacteria are critical for optimal muscle function: a potential link with glucose homeostasis. Am. J. Physiol. Metab. 317, E158-E171.

Nelke, C., Dziewas, R., Minnerup, J., Meuth, S. G., and Ruck, T. (2019). Skeletal muscle as potential central link between sarcopenia and immune senescence. EBioMedicine 49, 381-388. doi: 10.1016/j.ebiom.2019.10.034

Neto, J. V., De Melo, C. M., Maria, S., and Ribeiro, L. (2013). Effects of three-month intake of synbiotic on inflammation and body composition in the elderly: a pilot study. Nutrients 5, 1276-1286. doi: 10.3390/nu5041276

Ni Lochlainn, M., Nessa, A., Sheedy, A., Horsfall, R., García, M. P., Hart, D., et al. (2021). The PROMOTe study: targeting the gut microbiome with prebiotics to overcome age-related anabolic resistance: protocol for a doubleblinded, randomised, placebo-controlled trial. BMC Geriatr. 21:407. doi: 10. 1186/s12877-021-02301-y

Ni, Y., Yang, X., Zheng, L., Wang, Z., Wu, L., Jiang, J., et al. (2019). Lactobacillus and bifidobacterium improves physiological function and cognitive ability in aged mice by the regulation of gut microbiota. Mol. Nutr. Food Res. 63:1900603. doi: 10.1002/mnfr.201900603

Nilsson, N. E., Kotarsky, K., and Owman, C. (2003). Identification of a free fatty acid receptor, FFA2R, expressed on leukocytes and activated by short-chain fatty acids. Biochem. Biophys. Res. Commun. 303, 1047-1052. doi: 10.1016/s0006$291 \mathrm{x}(03) 00488-1$

Odamaki, T., Kato, K., Sugahara, H., Hashikura, N., Takahashi, S., Xiao, J., et al. (2016). Age-related changes in gut microbiota composition from newborn to centenarian: a cross-sectional study. BMC Microbiol. 16:90. doi: 10.1186/ s12866-016-0708-5

Okamoto, T., Morino, K., Ugi, S., Nakagawa, F., Lemecha, M., Ida, S., et al. (2019). Microbiome potentiates endurance exercise through intestinal acetate production. Am. J. Physiol. Metab. 316, 956-966.

Phillips, B. E., Atherton, P. J., Varadhan, K., Limb, M. C., Wilkinson, D. J., Sjøberg, K. A., et al. (2015). The effects of resistance exercise training on macro- and micro-circulatory responses to feeding and skeletal muscle protein anabolism in older men. J. Physiol. 12, 2721-2734. doi: 10.1113/jp270343

Picca, A., Ponziani, F. R., Calvani, R., Marini, F., Biancolillo, A., Gervasoni, J., et al. (2020). Gut microbial, inflammatory and metabolic signatures in older people with physical frailty and sarcopenia: results from the BIOSPHERE study. Nutrients 12:65. doi: 10.3390/nu12010065

Ponziani, R. F., Picca, A., Marzetti, E., Calvani, R., Conta, G., Del, F., et al. (2021). Characterization of the gut-liver-muscle axis in cirrhotic patients with sarcopenia. Liver Int. 41, 1320-1334. doi: 10.1111/liv.14876

Przewłócka, K., Folwarski, M., Kazmierczak-Siedlecka, K., Skonieczna-Zydecka, K., and Kaczor, J. J. (2020). Gut-Muscle axis exists and may affect skeletal muscle adaptation to training. Nutrients 12:1451.

Qian, F., Wang, X., Zhang, L., Chen, S., Allore, H., Bockenstedt, L., et al. (2012). Age-associated elevation in TLR5 leads to increased inflammatory 
responses in the elderly. Aging Cell 11, 104-110. doi: 10.1111/j.1474-9726.2011. 00759.x

Qiu, Y., Yu, J., Li, Y., Yang, F., Yu, H., Xue, M., et al. (2021). Depletion of gut microbiota induces skeletal muscle atrophy by FXR-FGF15/19 signalling. Ann. Med. 53, 508-522. doi: 10.1080/07853890.2021.1900593

Quiroga, R., Nistal, E., Estébanez, B., Porras, D., Juárez-fernández, M., Martínezflórez, S., et al. (2020). Exercise training modulates the gut microbiota profile and impairs inflammatory signaling pathways in obese children. Exp. Mol. Med. 52, 1048-1061. doi: 10.1038/s12276-020-0459-0

Rebello, C. J., Burton, J., Heiman, M., and Greenway, F. L. (2016). Gastrointestinal microbiome modulator improves glucose tolerance in overweight and obese subjects: a randomized controlled pilot trial. J. Diab. Compl. 29, 1272-1276. doi: 10.1016/j.jdiacomp.2015.08.023

Ríos-Covián, D., Ruas-Madiedo, P., Margolles, A., Gueimonde, M., De los ReyesGavilán, C. G., Salazar, N., et al. (2016). Intestinal short chain fatty acids and their link with diet and human health. Front. Microbiol. 7:185. doi: 10.3389/ fmicb.2016.00185

Rosenberg, I. H. (1997). Sarcopenia: origins and clinical relevance. J. Nutr. 127, 990S-991S.

Sakuma, K., and Yamaguchi, A. (2012). Sarcopenia and age-related endocrine function. Int. J. Endocrinol. 2012:127362.

Scheiman, J., Luber, J. M., Chavkin, T. A., Macdonald, T., Tung, A., Pham, L., et al. (2019). Meta-omics analysis of elite athletes identifies a performance-enhancing microbe that functions via lactate metabolism. Nat. Med. 25, 1104-1109. doi: 10.1038/s41591-019-0485-4

Schieber, A. M. P., Lee, Y. M., Chang, M. W., Collins, B., Downes, M., Evans, R. M., et al. (2015). Disease tolerance mediated by commensal E. coli via inflammasome and igf-1 signaling. Science 350, 558-563. doi: 10.1126/science. aac6468

Shafiee, G., Keshtkar, A., Soltani, A., Ahadi, Z., Larijani, B., Heshmat, R., et al. (2017). Prevalence of sarcopenia in the world: a systematic review and meta-analysis of general population studies. J. Diab. Metab. Disord. $16: 21$.

Shou, J., Chen, P. J., and Xiao, W. H. (2020). Mechanism of increased risk of insulin resistance in aging skeletal muscle. Diab. Metab. Syndr. 12:14.

Siddharth, J., Chakrabarti, A., Pannérec, A., Karaz, S., Rivron, D. M., Masoodi, M., et al. (2017). Aging and sarcopenia associate with specific interactions between gut microbes, serum biomarkers and host physiology in rats. Aging 9, 1698-1714. doi: 10.18632/aging.101262

Snijders, T., Nederveen, J. P., Mckay, B. R., Joanisse, S., Verdijk, L. B., Van Loon, L. J. C., et al. (2015). Satellite cells in human skeletal muscle plasticity. Front. Physiol. 6:683. doi: 10.3389/fphys.2015.00283

Soendergaard, C., Kvist, P. H., Thygesen, P., Reslow, M., Nielsen, O. H., Kopchick, J. J., et al. (2017). Characterization of growth hormone resistance in experimental and ulcerative colitis. Int. J. Mol. Sci. 18:2046. doi: 10.3390/ ijms 18102046

Sovran, B., Hugenholtz, F., Elderman, M., Van Beek, A. A., Graversen, K., Huijskes, M., et al. (2019). Age associated impairment of the mucus barrier function is associated with profound changes in microbiota and immunity. Sci. Rep. 9:1437.

Stewart, C. J., Ajami, N. J., Brien, J. L. O., Hutchinson, D. S., Daniel, P., Wong, M. C., et al. (2018). Temporal development of the gut microbiome in early childhood from the TEDDY study. Nature 562, 583-588.

Storelli, G., Defaye, A., Erkosar, B., Hols, P., and Royet, J. (2011). Lactobacillus plantarum promotes drosophila systemic growth by modulating hormonal signals through TOR-Dependent nutrient sensing. Cell Metab. 14, 403-414. doi: 10.1016/j.cmet.2011.07.012

Strasser, B., Wolters, M., Weyh, C., Krüger, K., and Ticinesi, A. (2021). The effects of lifestyle and diet on gut microbiota composition, inflammation and muscle performance in our aging society. Nutrients 13:2045. doi: 10.3390/nu130 62045

Taniguchi, H., Tanisawa, K., Sun, X., Kubo, T., and Hoshino, Y. (2018). Effects of short-term endurance exercise on gut microbiota in elderly men. Physiol. Rep. 6:e13935.

Theou, O., Jayanama, K., and Fernandez, J. (2019). Can a prebiotic formulation reduce frailty levels in older people? J. Frailty Aging 8, 48-52.

Thevaranjan, N., Puchta, A., Schulz, C., Verdu, E. F., Surette, M. G., Bowdish, D. M. E., et al. (2017). Age associated microbial dysbiosis promotes intestinal permeability, systemic inflammation, and macrophage dysfunction. Cell Host Microbe. 21, 455-466. doi: 10.1016/j.chom.2017.03.002

Ticinesi, A., Lauretani, F., Milani, C., Nouvenne, A., Tana, C., Del Rio, D., et al. (2017). Aging gut microbiota at the cross-road between nutrition, physical frailty, and sarcopenia: is there a gut- muscle axis? Nutrients 9:1303. doi: 10.3390/nu9121303

Ticinesi, A., Mancabelli, L., Tagliaferri, S., Nouvenne, A., Milani, C., Del Rio, D., et al. (2020). The gut-muscle axis in older subjects with low muscle mass and performance: a proof of concept study exploring fecal microbiota composition and function with shotgun metagenomics sequencing. Int. J. Mol. Sci. 21:8946. doi: 10.3390/ijms21238946

Ticinesi, A., Nouvenne, A., Cerundolo, N., Catania, P., Prati, B., Tana, C., et al. (2019). Gut microbiota, muscle mass and function in aging: a focus on physical frailty and sarcopenia. Nutrients 11:1633.

Tipton, K. D., Hamilton, D. L., and Gallagher, I. J. (2018). Assessing the role of muscle protein breakdown in response to nutrition and exercise in humans. Sport Med. 48, 53-64. doi: 10.1007/s40279-017-0845-5

Tran, L., and Greenwood-Van Meerveld, B. (2013). Age-Associated remodeling of the intestinal epithelial barrier. J. Gerontol. Biol. Sci. 68, 1045-1056. doi: 10.1093/gerona/glt106

Tsukuda, N., and Yahagi, K. (2021). Key bacterial taxa and metabolic pathways affecting gut short-chain fatty acid profiles in early life. ISME J. 15, 2574-2590. doi: 10.1038/s41396-021-00937-7

Valentino, T. R., Vechetti, I. J., Mobley, B. C., Dungan, C. M., Golden, L., Goh, J., et al. (2021). Dysbiosis of the gut microbiome impairs mouse skeletal muscle adaptation to exercise. J. Physiol. 1-39. doi: 10.1113/JP281788

van der Beek, C. M., Canfora, E. E., Kip, A. M., Gorissen, S. H. M., Olde, S. W. M., Van Eijk, H. M., et al. (2018). The prebiotic inulin improves substrate metabolism and promotes short-chain fatty acid production in overweight to obese men. Metabolism 87, 25-35. doi: 10.1016/j.metabol.2018.06.009

Van Tongeren, S. P., Slaets, J. P. J., Harmsen, H. J. M., and Welling, G. W. (2005). Fecal microbiota composition and frailty. Appl. Environ. Microbiol. 71, 6438-4262. doi: 10.1128/aem.71.10.6438-6442.2005

Verdi, S., Jackson, M. A., Beaumont, M., Bowyer, R. C. E., Bell, J. T., Spector, T. D., et al. (2018). An investigation into physical frailty as a link between the gut microbiome and cognitive health. Front. Aging Neurosci. 10:398. doi: 10.3389/fnagi.2018.00398

von Haehling, S., Morley, J. E., and Anker, S. D. (2012). From muscle wasting to sarcopenia and myopenia: update 2012. J. Cachexia Sarcopenia Muscle 3, 213-217. doi: 10.1007/s13539-012-0089-z

Vulevic, J., Drakoularakou, A., Yaqoob, P., Tzortzis, G., and Gibson, G. R. (2008). Modulation of the fecal microflora profile and immune function by a novel trans -galactooligosaccharide mixture (B-GOS) in healthy. Am. J. Clin. Nutr. 88, 1438-1446.

Walsh, M. E., Bhattacharya, A., Qaisar, R., Sloane, L., Rahman, M., Kinter, M., et al. (2015). The histone deacetylase inhibitor butyrate improves metabolism and reduces muscle atrophy during aging. Aging Cell 14, 957-970. doi: 10.1111/ acel. 12387

Walther, B., Lett, A. M., Bordoni, A., Tomás-cobos, L., Nieto, J. A., Dupont, D., et al. (2019). GutSelf: interindividual variability in the processing of dietary compounds by the human gastrointestinal tract. Mol. Nutr. Food Res. 63:1900677. doi: 10.1002/mnfr.201900677

Wang, S. Z., Yu, Y. J., and Adeli, K. (2020). Role of gut microbiota in neuroendocrine regulation of carbohydrate and lipid metabolism via the microbiota-gut-brain-liver axis. Microorganisms 8, 8-10.

Xiao, S., Fei, N., Pang, X., Shen, J., Wang, L., Zhang, B., et al. (2014). A gut microbiota-targeted dietary intervention for amelioration of chronic inflammation underlying metabolic syndrome. FEMS Microbiol Ecol. 87, 357367. doi: 10.1111/1574-6941.12228

Yamashita, H., Maruta, H., Jozuka, M., Kimura, R., Yamato, M., Saito, T., et al. (2014). Effects of acetate on lipid metabolism in muscles and adipose tissues of Type 2 diabetic otsuka long-evans tokushima fatty (OLETF) rats. Biosci. Biotechnol. Biochem. 8451, 2-9.

Yan, H., Diao, H., Xiao, Y., Li, W., Yu, B., He, J., et al. (2016). Gut microbiota can transfer fiber characteristics and lipid metabolic profiles of skeletal muscle from pigs to germ-free mice. Sci. Rep. 6:31786.

Yan, J., and Charles, J. F. (2018). Gut Microbiota and IGF-1. Calcif. Tissue Int. 102, 406-414. doi: 10.1007/s00223-018-0395-3 
Yatsunenko, T., Rey, F. E., Manary, M. J., Trehan, I., Dominguez-Bello, M. G., Contreras, M., et al. (2012). Human gut microbiome viewed across age and geography. Nature 486, 222-227. doi: 10.1038/nature11053

Yuan, X., Zhou, Y., Wang, W., Li, J., Xie, G., Zhao, Y., et al. (2013). Activation of TLR4 signaling promotes gastric cancer progression by inducing mitochondrial ROS production. Cell Death Dis. 4:e794. doi: 10.1038/cddis.20 13.334

Zheng, D., Liwinski, T., and Elinav, E. (2020). Interaction between microbiota and immunity in health and disease. Cell Res. 30, 492-506. doi: 10.1038/s41422020-0332-7

Zheng, Y., Song, Y., Han, Q., Liu, W., Xu, J., Yu, Z., et al. (2018). Intestinal epithelial cell-specific IGF1 promotes the expansion of intestinal stem cells during epithelial regeneration and functions on the intestinal immune homeostasis. Am. J. Physiol. Metab. 315, E638-E649.

Zhou, H., Yu, B., Sun, J., Liu, Z., Chen, H., Ge, L., et al. (2021). Short-chain fatty acids can improve lipid and glucose metabolism independently of the pig gut microbiota. J. Anim. Sci. Biotechnol. 12:61.
Conflict of Interest: The authors declare that the research was conducted in the absence of any commercial or financial relationships that could be construed as a potential conflict of interest.

Publisher's Note: All claims expressed in this article are solely those of the authors and do not necessarily represent those of their affiliated organizations, or those of the publisher, the editors and the reviewers. Any product that may be evaluated in this article, or claim that may be made by its manufacturer, is not guaranteed or endorsed by the publisher.

Copyright $\odot 2021$ Prokopidis, Chambers, Ni Lochlainn and Witard. This is an openaccess article distributed under the terms of the Creative Commons Attribution License (CC BY). The use, distribution or reproduction in other forums is permitted, provided the original author(s) and the copyright owner(s) are credited and that the original publication in this journal is cited, in accordance with accepted academic practice. No use, distribution or reproduction is permitted which does not comply with these terms. 